\title{
The future ain't what it used to be
}

\section{Strengthening the case for mutable futurism}

\section{Giacomo Andreoletti ${ }^{1}$ (i) $\cdot$ Giuseppe Spolaore ${ }^{2}$ (i)}

Received: 1 March 2021 / Accepted: 10 June 2021 / Published online: 14 July 2021

(c) The Author(s) 2021

\begin{abstract}
This paper explores mutable futurism, the view according to which the future can literally change - that is, it can happen that a future time $t$ changes from containing an event $E$ to lacking it (or vice versa). Mutable futurism has received little attention so far, and the details and implications of the view are underexplored in the literature. For instance, it currently lacks a precise metaphysical model and a formal semantics. Although we do not endorse mutable futurism, our goal here is to strengthen the case for mutable futurism and help establish it as a worthy contender in the debate on the philosophy of time. To attain this goal, (i) we try to make mutable futurism, along with its metaphysical and inferential commitments, as clear as possible, by providing it with a coherent metaphysical model and a plausible semantics, and (ii) we show that it can be backed by theoretical reasons.
\end{abstract}

Keywords Time $\cdot$ Future $\cdot$ Change $\cdot$ A-theory $\cdot$ Time travel $\cdot$ Free will

\section{Introduction}

There is a view in the philosophy of time that has received little attention so far. The view goes under the name of mutable futurism. According to mutable futurism, the future can change. This is not to say that things can change in the future. To say that things will change in the future is to say that at least two future times are qualitatively different. For instance, the year 2027 will contain different events than the year 2037

We owe the title to a famous quote by the American baseball player Yogi Berra.

$凶$ Giuseppe Spolaore

giuseppe.spolaore@unipd.it

Giacomo Andreoletti

giacomo.andreoletti@protonmail.com

1 University of Tyumen, Ulitsa Volodarskogo 6, Tyumen, Russian Federation

2 University of Padua, P.zza Capitaniato 3, Padova, Italy 
will. This kind of change in the future is an uninteresting platitude everyone agrees on. Nor is mutable futurism the view that future times change by becoming present, as a standard A-theorist would maintain. Rather, mutable futurism has it that the future is alterable and can literally change; that is, possibly, there are now events that will happen at a future time $t$, but later on, they will no longer be going to happen at $t$ (and may end up not happening at all). In other words, a future time $t$ can change from containing an event $E$ to not containing it, or vice versa.

Suppose that Anne is hiking on a mountain trail. At time $t_{1}$, she notices a stone rolling down slowly towards the mountain cliff. Usually she would ignore it. But now she feels strangely aware of the risk it could pose to other hikers. To prevent the fall, she would have to rush dangerously close to the cliff edge. At time $t_{2}$, after a moment of indecision, Anne throws herself ahead to intercept the stone. At time $t_{3}$, Anne is stretched on the ground and the stone is in her hand-and, luckily so. Anne realizes only now that Bob was just down the cliff on the stone's trajectory. The stone would have hit him. Let us assume that this is one of those cases where the future changes, if any such case is possible. A mutable futurist will claim that, from $t_{1}$ to $t_{2}$, the future time $t_{3}$ has changed. At $t_{1}, t_{3}$ features the event of Bob being hit by the stone. At $t_{2}$, this is no longer the case $-t_{3}$ now features Bob safely continuing his hike. This, in brief, is mutable futurism.

Mutable futurism was originally broached by Geach, who sketched it in his Providence and Evil (1977). More recently, Todd (2011, 2016) expanded on this sketch and provided new arguments for the view. Todd's conclusion is that mutable futurism should deserve the status of a theoretical contender in the metaphysical debates about time. So far, however, the view is still underexplored and underdeveloped. For instance, it lacks a metaphysical model and a semantics.

Although we are not mutable futurists ourselves, we do agree that mutable futurism is a view that deserves consideration. Here, our overarching goal is to strengthen the case for mutable futurism as a worthy contender among other theories of time, by making the position as clear as possible, showing that it can be made conceptually coherent, and explaining why certain philosophers might prefer it over its main contenders. To these aims, in what follows, (i) we offer a coherent metaphysical model for mutable futurism (section 2), (ii) we show that mutable futurism can be backed by theoretical reasons (section 3), and (iii) we define a plausible mutable futurists semantics (section 4).

\section{A metaphysical model for the mutable future}

Before offering any evidence for mutable futurism, it is important to address the issue of whether, and how, it can be made coherent. To this aim, we shall offer a consistent metaphysical model for mutable futurism, which also helps make some possible metaphysical costs of the position more precise.

According to mutable futurism, there can be events such that they will happen at a future time, and later on, will not happen at that future time. The future literally changes. Thus, it must be conceptually coherent to suppose that, for some times $t_{1}, t_{2}$ preceding another time $t_{3}$ (in symbols: $t_{1}<t_{2}$ and $t_{2}<t_{3}$ ): 
(1) At $t_{1}$, a certain event $E_{1}$ will obtain at $t_{3}$, but at $t_{2}, E_{1}$ will not obtain at $t_{3}$.

For instance, by mutable futurist standards, there is nothing conceptually incoherent in the following assumption:

(2) Today, a sea battle will happen tomorrow; but yesterday, no sea battle would be happening tomorrow.

An adequate metaphysical model for mutable futurism should enable us to make sense of claims like (1)-(2) in a coherent way.

Let us start by asking whether a mutable futurist model is to be couched in an A-theoretical or a B-theoretical framework. A-theorists believe in the existence of a moving objective present. In such a view, presentness is an absolute, non-perspectival feature of reality. The event of you reading this sentence now has the property of being objectively present. By the time you are reading this one, the event of you reading the previous sentence has moved into the past and is no longer objectively present. B-theorists deny the existence of such a feature of reality. We first explore whether a mutable futurist can adopt the B-theory. In particular, we look at a standard B-theory where time is one-dimensional.

The intuitive thought behind mutable futurism is that future actuality can possibly change when we move from a time to another. However, within the B-theory of time, it is not clear how the alleged relativization to times in (1) should be understood. For "at $t_{1}$ " cannot be a shorthand for "when $t_{1}$ is objectively present," simply because there is no objective present within the B-theory of time. Nor can a notion of subjective (or indexical) presentness be of any help here. A time $t$ is subjectively present relative to a time $t^{\prime}$ if and only if $t$ is identical to $t^{\prime}$ (see Spolaore and Torrengo 2019, $\S 2$, Spolaore and Gallina 2020,105). But if the expression "at $t_{1}$ " has to be interpreted as "when $t_{1}$ is identical to itself," then it does not pick any specific point in time, given that it is always the case that $t_{1}$ is identical to itself. If so, it is not clear when $t_{3}$ in (1) changes from featuring $E_{1}$ to not featuring it, especially if time is one-dimensional, leaving no room to relativize the claims in (1) to a second dimension of time. Thus, the alleged relativization to times must simply be dropped. We are then left with the following:

(3) A certain event $E_{1}$ will obtain at $t_{3}$, but $E_{1}$ will not obtain at $t_{3}$,

which is contradictory. The moral is that a standard B-theory with one-dimensional time is not a viable option for the mutable futurist.

Those familiar with the debate on the possibility of past-changing time travel will recognize a pattern here. It is tempting to think that, if we had time machines, we could take advantage of them to travel back into the past and change it. Tim might sit next to his time machine and plan to go back in time to kill baby Hitler. Tim thinks that he just needs to buy a gun and find a time when the baby is unprotected. It does not take much to kill an unprotected baby. However, upon philosophical reflection, this turns out to be a problematic task. Say that $t_{2}$ is the time when Tim devises his plan, $t_{3}$ is when he leaves with his time machine and $t_{1}$ is some time in 1890 soon after Hitler's birth. Tim travels from $t_{3}$ to $t_{1}$ and kills baby Hitler, thereby changing the past. The problem is that, if this occurs, the past of $t_{2}$ both contains Tim killing baby Hitler and does not contain it (Tim goes back in time because baby Hitler was not killed and grew up to 
commit the atrocious deeds that Tim wanted to prevent). Contradiction. Hence, time travelers cannot change the past, at least in a B-theoretic one-dimensional time (see Lewis 1976). However, the recent literature on time travel shows that we can resort to models of time that depart from a standard B-theory and make room for time travel that does result in alterations of the past. The strategy we have in mind is arguing that these models of past-alteration can be borrowed by the mutable futurist to model an alterable future. Here, we will briefly consider three of them: an hyper-eternalist model (discussed in Hudson and Wasserman 2010 and Wasserman 2017), a two-dimensional presentist model (Hudson and Wasserman 2010), and a hyper-presentist model (Law 2019). All of them adopt the A-theory of time and take time to be two-dimensional: reality unfolds across a first standard dimension of time and a second dimension of time called hyper-time.

In the hyper-eternalist model, the second dimension of time is cashed out in an eternalist manner (hyper-past, hyper-present and hyper-future times all exist); the same goes for ordinary time. The view can be put pictorially as in Fig. 1. The X-axis represents time, whereas the $y$-axis represents hyper-time. At each hyper-time, there is an eternalist block comprising all times. The A-theoretic objective present moves diagonally along the two-dimensional plane at the rate of one time unit per one hypertime unit, and change in general is understood as variation across time or hyper-time. Past times are composed of hyper-temporal parts, and hence, past alteration due to time travel is understood as hyper-variation across hyper-time. Thus, baby Hitler is alive and Tim is not around at the pair $\left(t_{1}, H t_{1}\right)$, whereas Tim kills baby Hitler at the pair $\left(t_{1}, H t_{3}\right)$. The past time $t_{1}$ changes from containing baby Hitler alive, when $\left(t_{2}, H t_{2}\right)$ is objectively present, to containing baby Hitler dead, when $\left(t_{3}, \mathrm{Ht}_{3}\right)$ is objectively present. Contradictions are avoided.

In the two-dimensional presentist model, the second dimension of time is still understood in an eternalist manner (all hyper-times exist). The difference here is that, at each hyper-time $H t$, we do not find a whole eternalist block, but rather only one time-slice-the one that is objectively present at $\mathrm{Ht}$. In this view, what was the case and what will be the case are described via fundamental tense operators that operate on present-tense propositions. In the case of time travel, what was the case can change relative to different hyper-times - not only in the sense that what was the case increases as the present moves forward but also in the sense that past facts themselves can change. So, when the $t_{2}$ slice is objectively present (at $H t_{2}$ ), it was the case, one unit of time before, that (baby Hitler is alive). When the $t_{3}$ slice is present (at $H t_{3}$ ), however, it was the case, two units of time before, that (baby Hitler is dead). The past changes insofar as there is the right sort of change with respect to what was the case.

In Law's hyper-presentist model, the second dimension of time is understood in a presentist manner, whereas ordinary time is eternalist time. In such a view, only hyperpresent eternalist blocks exist. So, when $\mathrm{Ht}_{2}$ is present, the eternalist block comprises all there is and features baby Hitler alive at $t_{1}$. When $\mathrm{Ht}_{3}$ is present though, the eternalist block hyper-presently features Tim killing baby Hitler at $t_{1}$. Here, the past is understood as enduring through hyper-time. And, changes in the past are explained by means of fundamental hyper-temporal operators - the one and the same enduring past loses or gains some events. In the example we are using, when $\mathrm{Ht}_{3}$ is objectively present, it is hyper-presently the case that (the past time $t_{1}$ features baby Hitler dead) and it hyper- 
was the case that (the past time $t_{1}$ features baby Hitler alive). By killing baby Hitler, Tim makes it the case that the past, as it hyper-presently is, is different from how it hyperused to be-it hyper-presently features baby Hitler's killing, while it did not hyperfeature it before. ${ }^{1}$ This detour into a mutable past has a bearing on our main focus, i.e. the mutable future. To start, note that when Tim kills baby Hitler, his act inevitably changes later parts of the timeline in various ways. For instance, World War II would not take place, or it would look extremely different. Our present would then look different too, and so would our future. Barring special cases when the alteration of the past does not produce any causal consequence or produces consequences that are limited in space and time, alterations of the A-theoretic objective past entail alterations of the objective future. Hence, everyone who accepts the possibility of changes in the past due to backward causation should likewise accept the possibility of changes in the future. Moreover, even if someone does not accept the physical or metaphysical possibility of time travel and backward causation, the past-alteration models are still instructive for the mutable futurist. In fact, those models of past alterations are easily applicable to a mutable future, even if someone wants to keep the past fixed. For instance, in the hyper-eternalist model, the mutable futurist claim in (1) can be understood as follows:

(4) When the pair $\left(t_{1}, H t_{1}\right)$ is objectively present, an event $E_{1}$ will obtain at $t_{3}$, but when the pair $\left(t_{2}, H t_{2}\right)$ is objectively present, $E_{1}$ will not obtain at $t_{3}$.

Within a two-dimensional presentist model, the claim in (1) is understood as follows:

(5) When $t_{1}$ is objectively present (at $H t_{1}$ ), it will be the case in two units of time that ( $E_{1}$ obtains). But when $t_{2}$ is objectively present (at $H t_{2}$ ), it will be the case in one unit of time that ( $E_{1}$ does not obtain).

Whereas in the hyper-presentist model, we have that:

(6) When $\mathrm{Ht}_{2}$ is objectively present, it is the case that hyper-presently (the future time $t_{3}$ does not feature $E_{1}$ ) and it hyper-was the case that (the future time $t_{3}$ features $\left.E_{1}\right)$.

Perhaps the hyper-presentist model is the one to be preferred, for it seems to be the one that fares better in capturing a strong sense of change understood in terms

\footnotetext{
${ }^{1}$ It is up for debate whether these models allow for genuine change, understood in terms of rewriting the past as opposed to merely affecting it. One could for instance argue that in what we are labeling the hypereternalist model, time travel results only in mere variation across the hyper-temporal dimension. That is, the time $t_{1}$ has distinct hyper-temporal parts that can qualitatively differ from each other. Tim is causally effective at the pair $\left(t_{1}, H t_{3}\right)$, insofar as he kills Baby Hitler there. But, so goes the objection, this should count at most as affecting the past, rather than changing it. From an external God's-eye perspective, the whole 2-dimensional set of events is permanent. Baby Hitler is alive at the pair $\left(t_{1}, H t_{1}\right)$, whereas he is not at the pair $\left(t_{1}, H t_{3}\right)$. One could reply though that change just $i s$ variation, and so the model captures the desired notion. Perhaps the most promising model among the three we briefly discussed is Law's hyper-eternalist model. In this account, there seems to be a robust sense of change, insofar as it is the enduring past itself that changes as hyper-time goes by. At any rate, it is an open issue in the literature whether A-theoretic two-dimensional models can really provide genuine change of the past. We do not even attempt to settle the issue here, as this would go beyond the scope of this paper. We just want to point out that these positions exist and that the mutable futurist can borrow from them to model her mutable future. For further discussion about the issue on whether a second dimension of time can allow for genuine change, see, among others, Meiland (1974), Goddu (2003), Bernstein (2017), and Pezet (2017).
} 
Fig. 1 A two-axis representation of the universe, where horizontal lines are eternalist blocks, the black portion of each line represents the objective past, and the edge of the black portion is the objective present

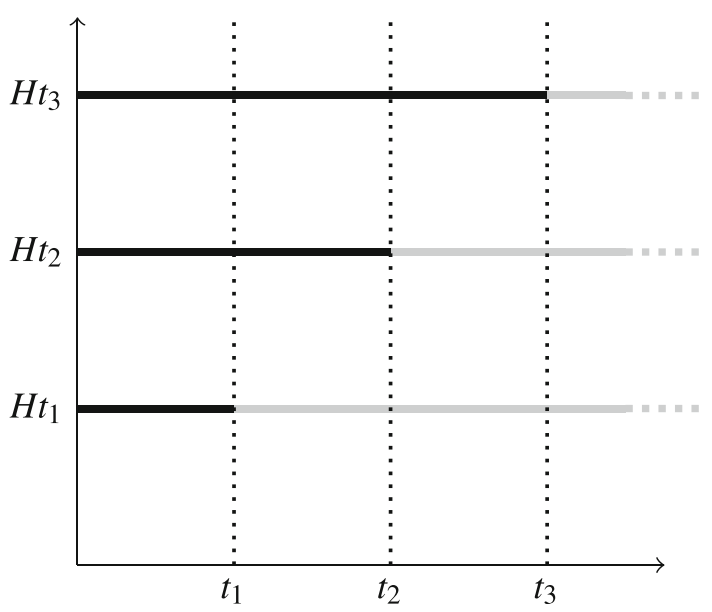

of rewriting (the past) or overwriting (the future). In fact, by adapting Law's pastchanging model to a future-changing model, we arguably end up with a robust notion of change in the future. The resulting picture would be one where what eternalist block exists changes as hyper-time goes by. In the example, the future time $t_{3}$ would be understood as an object that endures through hyper-time and possibly changes as hyper-time marches on. When $\mathrm{Ht}_{2}$ is objectively present, the future time $t_{3}$ is such that it hyper-presently does not feature $E_{3}$, whereas it hyper-used to be the case that the future time $t_{3}$ features $E_{3}$. However, we are not here to recommend one model over another; the final choice will depend on preferences and various considerations over the possibilities offered by the different theories of time. The point we want to stress is that, by drawing from the above models of past alterations, the mutable futurist ends up having at least three ways to coherently provide a metaphysical model for her view.

Before wrapping up this section, let us note two things. First, the three possibilities above are not exhaustive. There can probably be further ways of coherently modeling a mutable future. For instance, one could remove the objectively future sections of the timelines in the hyper-eternalist model, claim that there is an ersatz future made of future-oriented tensed facts that changes as time passes, and end up with a twodimensional growing block view where the ersatz future is mutable. Or, one could remove the second dimension of time from the two-dimensional presentist model, and claim that what will be the case changes in the relevant way as time passes. The set of future-oriented claims relative to any time $t$ would be internally consistent, but the combination of different sets might not be. Although it would be interesting to explore all possibilities, we do not attempt to give a complete taxonomy, given that we are here interested in arguing that mutable futurism can be metaphysically modeled.

Second, all viable options considered thus far feature an A-theoretic moving objective present. Is this just a coincidence? As shown above, mutable futurism cannot be cashed out in a standard B-theory. However, it is possible to remove the objective present from the hyper-eternalist model and end up with a two-dimensional B-theoretic model with different events at different hyper-times. Is such a non-standard B-theoretic model compatible with mutable futurism? 
There seem to be reasons to answer this question in the negative. Baron (2017) offers an argument against changing the past in two-dimensional B-theoretic models. In such models, Baron argues, one can at most affect the past, but there is no genuine change understood as rewriting or overwriting. If Baron is right, then the same considerations apply to the possibility of changing the future: Unless an A-theoretic model is adopted, there is no way of making sense of the future being rewritten or overwritten as time passes. If so, then mutable futurism not only naturally matches with the A-theory but also requires it.

To sum up, mutable futurism appears to be contradictory at first sight. For it claims, for instance, that there are future events that will happen at a later time $t$, but later on will no longer be going to happen at $t$. And certainly, the view $i s$ contradictory if spelled out within a standard B-theoretical framework. Yet, as we have shown, the view can be made metaphysically coherent by resorting to other models of time. Probably, most philosophers favor the B-theory over the A-theory of time. Certainly, positing the existence of a second dimension of time, as the three models described above do, is a cost insofar as it inflates our ontology. Yet, our overarching goal here is to argue that mutable futurism deserves the role of a theoretical contender. The fact that it can be provided with coherent metaphysical models is a first step in that direction.

\section{Theoretical reasons for mutable futurism}

Our main goal in this paper is to help establish mutable futurism as a worthy contender in the debate about the nature of time. In the previous section, we have countered the idea that mutable futurism is contradictory and, thus, a non-starter. On the contrary, as we have shown, mutable futurism can be provided with a coherent metaphysical model. Moreover, in the next section, we offer a semantics for the kind of claims that mutable futurists wish to make. Yet, for mutable futurism to earn its keep, it is not enough to show that it occupies a consistent spot in the logical space and that it can have its own semantics. One question needs still to be answered: What kind of theoretical reasons can one have to endorse mutable futurism?

Geach (1977) observes that we need a mutable future to make sense of the notion of prevention. For, when someone prevents something, she prevents something that was going to happen but, ultimately, does not happen thanks to the preventative act. Geach argues that analyzing the notion of prevention by saying that what is prevented is something that was going to happen unless someone prevents it would be circular. ${ }^{2}$ Therefore, a mutable future is needed to provide an analysis of prevention.

Todd (2016) argues that mutable futurism can provide an original answer to the problem of logical fatalism, as well as a solution to a puzzle about the utility of foreknowledge. As for logical fatalism, mutable futurism gives a principled answer to the master argument for fatalism. The argument is notoriously based on the principle that, if it is true now that an event $E$ will obtain, then it is historically necessary that $E$ will obtain. But within mutable futurism, this principle is invalid, for even if it is now

\footnotetext{
2 Todd (2016, 2081-85) considers, and rejects, a non-circular counterfactual analysis of prevention, according to which $X$ prevents $Y$ if and only if (i) $X$ is causally sufficient for not- $Y$, and (ii) had $X$ not occurred, $Y$ would have occurred.
} 
Fig. 2 Events in a mutable futurist scenario per the hyper-eternalist model

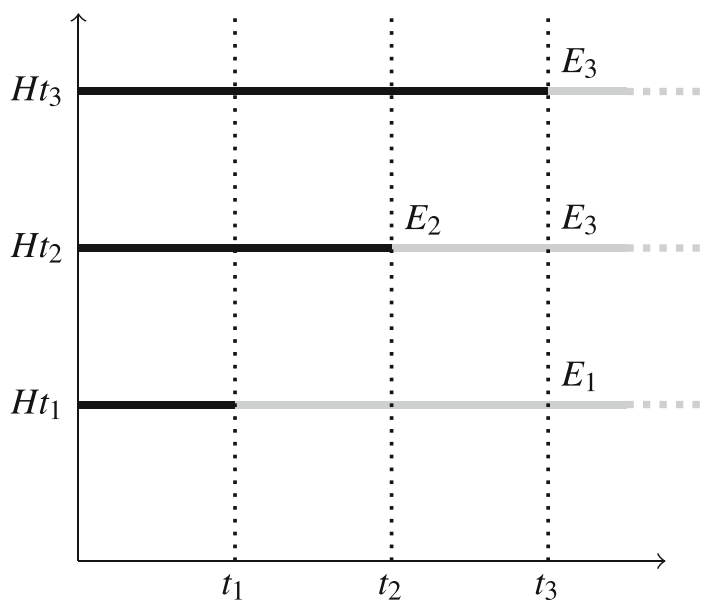

true that $E$ will obtain, something happening in the interim might change the future and prevent $E$ from happening (see also Andreoletti 2019 about this point). As for the puzzle about foreknowledge, Todd observes that we naturally take foreknowledge to be useful (e.g., knowledge about the future stock market trends), and it appears that, the more foreknowledge we have, the better. However, if the future is unchangeable having total foreknowledge is completely useless, for we cannot alter what we know will be the case (e.g., which stocks we will buy, perhaps those that will perform bad in the future). But, if we can change the future, foreknowledge turns out to be always useful, as intuitively it should be.

In this section, we try to provide some further reasons why someone might be interested in mutable futurism. Our general strategy is as follows. We argued before that mutable futurism requires an A-theoretic objective present. Some prefer the Atheory over the B-theory. We argue that if someone goes A-theoretic, then there are reasons to believe in mutable futurism, or at least to regard it as a respectable option.

Recall the mutable futurist picture (see Figure 2). When $t_{1}$ is objectively present, an event $E_{1}$ will happen at $t_{3} .{ }^{3}$ Yet, when $t_{2}$ becomes present, something occurring at $t_{2}$, say an event $E_{2}$, makes the future different from how it used to be. Thanks to $E_{2}$ 's occurrence, the future time $t_{3}$ no longer features $E_{1}$ but now features $E_{3}$ instead. Thus, a mutable futurist believes in the occurrence of events, such as $E_{2}$ and $E_{3}$, that obtain even though they were not in the future of some past time. For lack of a better word, let us call these events newcomers.

Newcomers: An event $E$ is a newcomer if and only if (a) $E$ occurs at some objectively present time $t$ and (b) it was not the case, for every time $t^{\prime}$ such that $t^{\prime}<t$, that $E$ was in the future of $t^{\prime}$ when $t^{\prime}$ was objectively present.

The occurrence of newcomers is not a peculiar feature of mutable futurism. Consider the following passage from Prior (1996):

\footnotetext{
3 This kind of claims should in principle be more thoroughly specified, depending on which one of the three models discussed in section 2 one decides to adopt; hereafter, for simplicity, we shall speak freely of a time being objectively present.
} 
One of the big differences between the past and the future is that once something has become past, it is, as it were, out of our reach - once a thing has happened, nothing we can do can make it not to have happened. But the future is to some extent, even though it is only to a very small extent, something we can make for ourselves. [...] In my own logic with tenses I would express it this way: we can lay it down as a law that whatever now is the case will always have been the case; but we can't interchange past and future here and lay it down that whatever now is the case has always been going to be the case - I don't think that's a logical law at all; for if something is the work of a free agent, then it wasn't going to be the case until that agent decided that it was.

Prior hints at the idea that genuinely free acts are an instance of what we call newcomers. Newcomers other than free acts, so understood, can be envisaged. For instance, if someone believes that the world features some kind of objective indeterminacy, then she is likely to believe in the occurrence of events that happen and were not going to happen-for instance, because they were not settled by their past. Similarly, if someone interprets " $E$ will happen" as "it is presently determined that it will be the case that $E$," we might then have that a present event $E$ occurs, although at some past time, it was not the case that $E$ would happen. Thus, a mutable futurist is not alone in allowing for the occurrence of newcomers. ${ }^{4}$

The hallmark of mutable futurism is something else. In the example above, $E_{1}$ is in the future when $t_{1}$ is objectively present. However, a newcomer comes by at $t_{2}$ and makes the future change from featuring $E_{1}$ to featuring $E_{3}$. Looking retrospectively from $t_{3}$, a mutable futurist maintains that, when $t_{1}$ was present, $E_{1}$ would happen at $t_{3}$ but, when $t_{2}$ was present, it was not the case that $E_{1}$ would happen at $t_{3}$. Thus, a mutable futurist believes in events, such as $E_{1}$, that used to be future in the past, but as can be appreciated retrospectively, never found their way into the present. Let us call these events bygones.

Bygones: An event $E$ is a bygone if and only if (a) $E$ does not occur at some objectively present time $t$, and (b) there is a time $t^{\prime}$ such that $t^{\prime}<_{n} t$ (i.e., $t^{\prime}$ is $n$ time units earlier than $t$ ) and $E$ was $n$ time units in the future of $t^{\prime}$ when $t^{\prime}$ was objectively present.

Positing the existence of bygones entails denying (a possible interpretation of) the principle that having been in the future entails being present some time hence. The principle is highly plausible, and rejecting it is admittedly a hard pill to swallow. ${ }^{5}$ This could be why, whereas newcomers are sometimes posited, bygones are nowhere to be found in other theories of time. However, we take it that, if an A-theorist accepts

\footnotetext{
${ }^{4}$ It also seems that any position inconsistent with the principle that Todd and Rabern $(2021,103)$ call retro-closure ("from the fact that something is true, it follows that it was the case that it would be true") makes room for newcomers.

5 However, the hardness of the pill is not to be overestimated. First, recall that we are operating in a Atheoretical dialectical context, and that most A-theorists reject the converse of this principle (viz., the view that not being in the future entails not being present some time hence), which is very appealing as well. Second, we believe that some philosophers tend to exaggerate the impact of giving up the principle. For instance, according to Pezet (2017), "if ... being future does not entail being present some time hence, then I simply do not know what content is left in these notions. After all, the reasons we care about past and future is precisely because they were or will be present" (21). Agreed, the mutable futurist must deny that
} 
newcomers based on the kind of libertarian considerations expressed by Prior in the above passage, she should also be open to bygones. (This is not to say that she is forced to accept them - rather, she should regard the acceptance of bygones as a viable theoretical option.) And, if someone accepts both newcomers and bygones, then the resulting picture is a mutable futurist one.

Suppose someone believes, following Prior, that genuinely free acts are newcomers. A family of theories within the libertarian approach to free will, viz. theories of agent causation, seem to incarnate this idea. Theories of agent causation rest on the assumption that events are not always caused by other events. Agents can have causal powers too. In general terms, agent causalists hold that when an agent acts freelywith the kind of free will relevant for moral responsibility - the agent herself does the causing, viz. the agent stands in a causal relation with the events that she makes happen. Moreover, agent causalists hold that, when an agent exercises her power of agent causation, she is in some significant sense the ultimate causal source, i.e., either because no prior event whatsoever is causing the agent to exercise the power of agent causation or because prior events do not necessitate the agent to exercise her power in the way she actually does. To use the famous Aristotelian example, a man (an agent) causes the event of the hand being moved, which in turn causes the event of the staff being moved, which in turn causes the rock to move, and so on. What is crucial is that the agent initiates a causal chain of events, where the initial step is not necessitated by prior events but is instead "the work of a free agent."

The theory of agent causation comes in different flavors. Versions of the theory are offered, among others, by Chisholm (1966), Clarke (1993), and O'Connor (1995, 2001) (see Griffith (2013. Ch.5) for a general overview). For our purposes, we can use the following general characterization of an agent-causalist free action, which arguably, many agent-causalists would accept (see Markosian 1999 for a discussion):

Free Action: An action A by agent $\mathrm{S}$ at $t$ is free if and only if (i) A is caused by $\mathrm{S}$, and (ii) $\mathrm{A}$ is not caused (or necessitated) by events outside of $\mathrm{S}$ (including events that are prior to $t$ ).

In such a view, agents play a significant role in initiating causal chains. Obviously, agents are not the only things endowed with causal powers. Events have causal powers too. Thus, this view admits a double causal order. On the one hand, we have an agentive causal order, which is set in motion by agents' free acts. On the other hand, we have a purely eventive causal order, which only consists of causal chains of events. Crucially, under the theory of agent causation, the agentive causal order is, to a certain extent, independent of the eventive order, insofar as the eventive order does not cause (or does not necessitate) what is freely caused by agents.

But how precisely do these different causal orders contribute to determine the future as a whole? Since the agent's free decisions enjoy a degree of independence from the eventive order, there is a picture that immediately suggests itself. The eventive order,

\section{Footnote 5 continued}

being future entails being present some time hence, for they accept bygones. However, the mutable futurist can have an alternative explanation for why we care about the future. If an unpleasant event $\mathrm{E}$ is currently in our future, we do care about it simply because, as things stand now, E will obtain - a claim which is perfectly compatible with mutable futurism. It is then up to us, and in our interest in case of unpleasant events, to act in such a way that the event will become a bygone. 
so the picture goes, is potentially in a position to determine the whole of the future, and absent any agentive intervention, it does so. Thus, the future is populated by events that follow the eventive causal order. Those events are to take place, unless certain agents freely prevent them from happening. When they do so, agents 'overwrite' the output of the eventive order, and modify the corresponding course of events. The things responsible for the overwriting are newcomers, while bygones are what get overwritten. As observed above, accepting both newcomers and bygones commits one to accepting mutable futurism.

To exemplify, recall the case from the outline about Anne deciding to intercept the stone falling from the cliff. When $t_{1}$ is objectively present, Anne notices the stone falling but she has not yet decided she would intervene to stop it. Later on, at $t_{2}$, she decides to intervene and intercept the stone. When $t_{3}$ is objectively present, Anne intercepts the stone and Bob is not hit. Say that when Anne decides to intervene, she is exercising her power of agent causation. Her free decision causes a series of subsequent events, such as Anne throwing herself to catch the stone and the stone actually ending up in her hands. This is the agentive causal order set in motion by Anne. What is relevant is that under the agent causalist view, Anne's decision to intercept the stone is not caused (or necessitated) by events prior to $t_{2}$. Anne starting the agentive causal chain by means of her power of agent causation is not yet in the future when $t_{1}$ is objectively present. But if so, what does the future of $t_{1}$ feature? It must be something other than Anne deciding to intervene to stop the stone, as that is a newcomer. A natural answer is that it features how things will go according to the eventive order up to $t_{1}$, viz. the stone fatally hitting Bob down the cliff at $t_{3}$. However, when $t_{2}$ becomes present, the newcomer of Anne deciding to intervene takes place and changes the future time $t_{3}$. As a result, Bob being hit by the stone never finds its way into the objective present. Thus, this event is a bygone.

To be explicit, we are not here saying that agent causalists or other libertarians must be committed to believing in bygones. The theory of agent causation is compatible with any theory of time that gives room to some form of indeterminism in the world. However, there is a difference between a certain philosophical view being compatible with an underlying metaphysical picture, and supporting it. From an agent-causalist viewpoint, by means of their free actions, agents make a distinctive difference with respect to how things will be. Moral responsibility hinges precisely on the kind of difference agents can make. In order to do full justice of this agent-causalist commitment, one has to have the resources to make perfect sense of what this difference consists in. Mutable futurists have the resources to do so, because they can represent the difference made by agents in terms of a difference between different futures. It is unclear whether the same holds for other indeterminist alternatives. Growing blockers deny the existence of the future, so they cannot resort to the same kind of explanation. Branching-time theorists do have the resources to represent the difference made by agents. However, there are doubts that they can adequately represent the contribution of the agent to those changes, in a way that makes for a reasonable picture of the agent's responsibility and moral commitments (see, e.g., Cameron 2015, 177-179).

To wrap up, agent causalist views seem to suggest the presence of a double causal order, viz. the agentive order set in motion by agents free acts and the eventive order. This double causal order not only brings into the picture newcomers, something which 
is accepted within many theories of time, but it also seems to naturally suggests the presence of bygones. And, as said, once one has both newcomers and bygones on board, then the result is a mutable future.

\section{Semantics}

In this section, we outline some mutable futurist semantics for a simple temporal language. The key idea behind our proposals is that tense and temporal adverbs can play (at least) two roles. First, they can be used to locate certain events in the timeline, as in "A sea battle is taking place now." Second, they can be used to select the hypertime from which the timeline is considered, as in "Now it is true that there will be a sea battle." If mutable futurism is correct, then it is important to take this second role into account, for a change in the hyper-time under consideration can make a difference in the temporal location of certain events.

Before introducing any formal definition, it is useful to provide an intuitive picture of our proposal. To this aim, we shall resort to a simple trick: we shall capitalize those expressions that are understood to pick up hyper-times as opposed to times. Thus, for instance, "Now" picks up the present hyper-time, as opposed to "now," which picks up the present time; "Yesterday" picks up yesterday's hyper-times, as opposed to "yesterday," which picks up yesterday's times; and so on. Similarly with tense: non-capitalized tensed expressions ("is," "was," "will," ...) operate on times, capitalized ones ("Is," "Was," "Will," ...), on hyper-times. With this in mind, consider the following sentences:

(7) Yesterday it Was true that there is a sea battle,

(8) yesterday there was a sea battle.

Despite their similarity, (7) and (8) mean very different things. While (7) says that, from yesterday's perspective, a sea battle is located now, (8) says that, from the present perspective, a sea battle is located yesterday.

In a similar way, the propositional tense language $\mathcal{L}$ we adopt encodes the distinction between times and hyper-times. It includes two standard, Priorean metric tense operators that operate on times, namely, was $[n]$ (which reads: "It was the case $n$ time units ago ") and will[ $n]$ ("It will be the case in $n$ time units"). In addition, $\mathcal{L}$ includes two capitalized, non-standard metric operators that operate on hyper-times, namely, Was $[n]$ ("It Was the case $n$ time units Ago") and Will[n] ("It Will be the case in $n$ time units"). Let us call these operators hyper-tense operators. Intuitively, hyper-tense operators shift the hyper-time from which the timeline is considered. As is usual in propositional tensed languages, atoms $p, q, \ldots$ are understood to express presenttensed statements like, "There is a sea battle" and "It rains." Thus, for instance-and adopting days as our unofficial time unit-we can formalize sentences (7) and (8) as follows:

(9) Was [1]p

(10) was [1]p 
In the notation of Backus-Nauer form (BNF; see, e.g., Goldblatt 1992, 3), sentences $\mathcal{A}$ of language $\mathcal{L}$ can be specified as follows, where $n$ is a non-negative number:

$$
\mathcal{A}::=p|\neg \mathcal{A}|(\mathcal{A} \wedge \mathcal{A}) \mid \text { was }[n] \mathcal{A}|\operatorname{Was}[n] \mathcal{A}| \text { will }[n] \mathcal{A} \mid \operatorname{Will}[n] \mathcal{A}
$$

To provide an adequate semantics for a metric language like $\mathcal{L}$, a metric must be defined on the frame. More specifically, we assume that, for any two times $t, t^{\prime}$, there exists a number $n$ that indicates the distance between $t$ and $t^{\prime}$ expressed in time units.

To evaluate sentences of language $\mathcal{L}$, which includes both tense and hypertense operators, two temporal indices are needed, one corresponding to a time and another corresponding to a hyper-time. We let a point be an ordered pair $\left(t, t^{\prime}\right)$ of times. When we speak of a sentence $\mathcal{A}$ being evaluated at a point $\left(t, t^{\prime}\right)$, we mean that $\mathcal{A}$ is evaluated relative to time $t$ and hyper-time $H t^{\prime}$ corresponding to time $t^{\prime}$. Sometimes, we shall also use $x, y \ldots$ as variables for points.

Now for the semantics. We shall start with models and semantic clauses that encode no limitation whatsoever over reference to future hyper-times, and in which bivalence holds unrestrictedly at any point. Then, we shall outline different ways to put constraints on our semantics, which many A-theorists will find natural.

A general mutable-futurist (GMF) frame $\mathfrak{F}$ is a tuple $(T, \leq, \Delta)$ where:

- $T$ is a nonempty set of times;

- the temporal precedence relation $\leq$ is an ordering on $T$. The relation $\geq$ of temporal succession is the inverse of $\leq$, and the strict orderings $<$ and $>$ are defined in the obvious way;

- $\Delta$ is a metric function from $T \times T$ onto the set of non-negative numbers (see Koymans 1990 for details). We write $t<_{n} t^{\prime}$ to indicate that $t<t^{\prime}$ and $\Delta\left(t, t^{\prime}\right)=n$.

A GMF model $\mathfrak{M}$ is a pair $(\mathfrak{F}, v)$ where $\mathfrak{F}$ is a GMF frame and $v$ is an interpretation function from atoms of $\mathcal{L}$ to points. We assume that $v$ is bivalent: any atom is definitely true or false at any point. This assumption is not entailed by mutable futurism, but it is natural enough and simplifies things. Moreover, we impose the following condition:

Past stability (PS): For any atom $p$ of $\mathcal{L}, x \in v(p) \Leftrightarrow y \in v(p)$ if $t=t_{1}$ and both $t \leq t^{\prime}$ and $t_{1} \leq t_{1}^{\prime}$.

Condition PS corresponds to the idea that the past does not change. It can be given up if also the past is taken to be mutable.

Now for the definition of truth in a GMF model at a point.

Definition 1 A sentence $\mathcal{A}$ is true at point $\left(t, t^{\prime}\right)$ in a GMF model $\mathfrak{M}$ (in symbols: $\mathfrak{M}, t, t^{\prime} \vDash \mathcal{A}$ ) if and only if:

- $\mathcal{A}$ is an atom $p \Rightarrow\left(t, t^{\prime}\right) \in v(p)$;

- $\mathcal{A}$ has form $\ulcorner\neg \mathcal{B}\urcorner \Rightarrow$ it is not the case that $\mathfrak{M}, t, t^{\prime} \vDash \mathcal{B}$;

- $\mathcal{A}$ has form $\ulcorner\mathcal{B} \wedge \mathcal{C}\urcorner \Rightarrow \mathfrak{M}, t, t^{\prime} \vDash \mathcal{B}$ and $\mathfrak{M}, t, t^{\prime} \vDash \mathcal{C}$;

- $\mathcal{A}$ has form $\ulcorner$ was $[n] \mathcal{B}\urcorner \Rightarrow \mathfrak{M}, t^{\prime \prime}, t^{\prime} \vDash \mathcal{B}$, with $t^{\prime \prime}<_{n} t$;

- $\mathcal{A}$ has form $\ulcorner$ will $[n] \mathcal{B}\urcorner \Rightarrow \mathfrak{M}, t^{\prime \prime}, t^{\prime} \vDash \mathcal{B}$, with $t<_{n} t^{\prime \prime}$;

- $\mathcal{A}$ has form $\ulcorner$ Was $[n] \mathcal{B}\urcorner \Rightarrow \mathfrak{M}, t, t^{\prime \prime} \vDash \mathcal{B}$, with $t^{\prime \prime}<{ }_{n} t^{\prime}$;

- $\mathcal{A}$ has form $\ulcorner$ Will $[n] \mathcal{B}\urcorner \Rightarrow \mathfrak{M}, t, t^{\prime \prime} \vDash \mathcal{B}$, with $t^{\prime}<_{n} t^{\prime \prime}$. 
Logical notions, such as GMF-logical truth or GMF-validity, are defined in the usual way. 6

This semantics satisfies a key mutable futurist desideratum: in GMF models, the future is mutable in the sense that a sentence $\mathcal{A}$ can be GMF-true (GMF-false) at a time $t$ relative to hyper-time $H t$ and GMF-false (GMF-true) at $t$ relative to a previous hyper-time $H t^{\prime}$. As a consequence, for instance, the following schemas are not GMF-valid:

(11) $\mathcal{A} \leftrightarrow \operatorname{Was}[n] \mathcal{A}(\mathcal{A}$ if and only if $n$ time units Ago it Was the case that [now] $\mathcal{A})$.

(12) will $[n] \mathcal{A} \leftrightarrow$ Will $[n]$ will $[n] \mathcal{A}$ (It will be the case in $n$ time units that $\mathcal{A}$ if and only if it Will be the case in $n$ time units that it will be the case in $n$ time units that $\mathcal{A})$.

If condition PS is accepted, the semantics does validate the mirror principles of (11)(12), thus preserving the asymmetry between (immutable) past and (mutable) future:

(13) $\mathcal{A} \leftrightarrow \operatorname{Will}[n] \mathcal{A}$

(14) $\operatorname{was}[n] \mathcal{A} \leftrightarrow$ Was $[n]$ was $[n] \mathcal{A}$.

Above, we argued that mutable futurism is naturally coupled with the A-theory. However, the semantics introduced thus far can raise some perplexities from an Atheoretical perspective, for at least two reasons. First, GMF models do not encode any information about the objectively present time. Second, every sentence receives a determinate GMF-truth-value relative to any point, and no limitation is imposed on what points can be referred to. As a result, for any future hyper-time $H t$, it is (tenselessly) determined what events populate the timeline from $H t$ 's perspective, no matter how far removed $\mathrm{Ht}$ is from the present time. But arguably, A-theoretic mutable futurists will hold that there can be changes in the future only when we move to a new hyper-time's perspective, and that, prior to that hyper-time, it is indeterminate what changes, if any, will occur. This is an especially natural view if one adopts the hyper-presentist model, where only the hyper-present timeline exists (see section 2).

To overcome the first perplexity, it is sufficient to define a more A-theoretically friendly kind of models than GMF models. To this aim, we let an A-mutable-futurist model (AMF) $\mathfrak{M}_{A}$ be a pair $\left(\mathfrak{M}, t_{\mathfrak{p}}\right)$ of a GMF model and a privileged time $t_{\mathfrak{p}}$, which is thought to be the objectively present time.

To overcome the second perplexity, different strategies are available. In what follows, we shall briefly outline two of them. We think of these strategies just as

\footnotetext{
${ }^{6}$ It is worth noting that, in the semantic clauses for the metric operators, times and hyper-times are shifted in an independent way. As a consequence, the numbers following tense operators do not 'sum up' with those following hyper-tense operators; e.g., Was [1] was[1] $p$ means that $p$ was true yesterday from Yesterday's perspective, not that $p$ was true the day before yesterday from Yesterday's perspective. Moreover, the relative order of consecutive operators is irrelevant; e.g., Was [1] will [3]Will [2] $p$ is equivalent to will [3]Will[2]Was [1]p.
} 
significant examples, with no pretension of exhaustivity. The first strategy is based on a simple idea-imposing constraints on AMF models to make reference to future hyper-times (viz., hyper-times following the privileged time) redundant. To this aim, we let the redundancy variant of an AMF model $\mathfrak{M}_{A}=\left((T, v), t_{\mathfrak{p}}\right)$ be a model $\left(\left(T, v^{\prime}\right), t_{\mathfrak{p}}\right)$ such that, for any atom $p$ and any point $\left(t, t^{\prime}\right)$, if $t \leq t_{\mathfrak{p}}$ then $\left(t, t^{\prime}\right) \in v^{\prime}(p) \Leftrightarrow\left(t, t^{\prime}\right) \in v(p)$, and $\left(t, t^{\prime}\right) \in v^{\prime}(p) \Leftrightarrow\left(t, t_{\mathfrak{p}}\right) \in v^{\prime}(p)$ otherwise. Intuitively, in the redundancy variant of an AMF model, we have that, from the perspective of any hyper-time following the 'present' hyper-time $H t_{\mathfrak{p}}$, the timeline looks exactly the same as it looks from the perspective of $H t_{\mathfrak{p}}$. Redundant truth in an AMF model $\mathfrak{M}_{A}$ is defined as truth in the redundancy variant of $\mathfrak{M}_{A}$. Moreover, a sentence $\mathcal{A}$ is said to be redundantly true simpliciter in $\mathfrak{M}_{A}=\left(\mathfrak{M}, t_{\mathfrak{p}}\right)$ if and only if $\mathcal{A}$ is redundantly true at $\left(t_{\mathfrak{p}}, t_{\mathfrak{p}}\right)$. The rationale behind this proposal is that the objectively present time provides us with the best possible perspective in assessing future-oriented sentences, assuming that facts obtaining at future hypertimes are not yet part of reality, and thus, they are epistemically and/or referentially inaccessible.

A problem with this semantics is that the following schema turns out to redundantly true simpliciter in all $\mathfrak{M}_{A}$ models:

$$
\operatorname{will}[n] p \leftrightarrow \operatorname{Will}\left[n^{\prime}\right] \text { will }[n] p
$$

As assessed at $\left(t_{\mathfrak{p}}, t_{\mathfrak{p}}\right)$, and ignoring metrics, (15) says that something is Now a future truth if and only if it Will remain a future truth, which intuitively entails that the future Will not change. If we define (redundant) validity as (redundant) truth simpliciter in all AMF models, (15) turns out to be valid-a result that is prima facie at odds with the key mutable futurist tenet that, possibly, the future Will change. Perhaps this is a problem that mutable futurists can solve or at least put into perspective. However, it motivates the quest for a different approach.

The second strategy is supervaluationist in spirit. Let a free-future variant of an AMF model $\mathfrak{M}_{A}=\left(\mathfrak{M}, t_{\mathfrak{p}}\right)$ be a model that coincides with $\mathfrak{M}_{A}$ except that, for any point $\left(t, t^{\prime}\right)$ such that $t, t^{\prime}>t_{\mathfrak{p}}$, and for any contingent atom $p$, the choice of whether $\left(t, t^{\prime}\right) \in v(p)$ is free. Intuitively, free-future variants represent all the possible ways the future might be. Free-future truth in an AMF model $\mathfrak{M}_{A}$ is defined as truth in all free-future variants of $\mathfrak{M}_{A}$. Moreover, we shall say that a sentence is free-future true simpliciter in $\mathfrak{M}_{A}$ if it is free-future true at $\left(t_{\mathfrak{p}}, t_{\mathfrak{p}}\right)$. Free-future validity is defined as free-future truth simpliciter in all models $\mathfrak{M}_{A}$.

This strategy escapes the previous problem, as (15) is free-future invalid. Indeed, the strategy aims to reconcile the mutable futurist tenet that the future can change with the view that the present perspective is metaphysically and epistemically privileged (what is true simpliciter is what is true now from the perspective of Now). All this, without violating the idea that the future is fully open, in the sense that there is no fact of the matter as to what will be true from the perspective of future hyper-times. Moreover, the strategy is flexible: we can impose different constraints on what atoms count as contingent and non-contingent. For instance, we can count as non-contingent all atoms representing stable or nomological truths or falsities, such as physical necessities or impossibilities. 


\section{Conclusion}

Our main goal in this paper was to strengthen the case for mutable futurism, a view that so far has received little attention in the philosophical debate about time. To this end, we showed how mutable futurism can be provided with a coherent metaphysical model and a plausible semantics. We also clarified the commitments of mutable futurism, its implications, and some of its analogies and differences with other positions in the debate about time. Moreover, we argued that some positions in the debate about free will should find mutable futurism attractive.

We take it that this work provides a comprehensive characterization of mutable futurism, which clarifies its nature, its main commitments and some key theoretical advantages over rival views. In our view, this should help mutable futurism be the focus of a sound debate, in which the risk of misunderstanding the view or exaggerating its consequences are kept to a minimum. If we are right, mutable futurism should now be ready to fully join the philosophical conversation on time. We also think that there are further ramifications of mutable futurism, and these are worth considering for instance, how does mutable futurism fare in the debate about how we should understand the asymmetry between the past and the future? At any rate, we leave those considerations for the (perhaps mutable) future.

Acknowledgements The authors want to thank Giuliano Torrengo, Roberto Loss, Ulrich Meyer, and two anonymous reviewers for their careful and valuable comments on previous versions of this paper.

Funding Open access funding provided by Universitá degli Studi di Padova within the CRUI-CARE Agreement. Giacomo Andreoletti's work was supported by the project PID2019-108762GB-I00 of the Spanish Ministry of Science and Innovation. Giuseppe Spolaore's work was supported by the project SPOL_BIRD2020_01 ("Epistemic virtues and knowledge") of the FISPPA Department, University of Padua.

Open Access This article is licensed under a Creative Commons Attribution 4.0 International License, which permits use, sharing, adaptation, distribution and reproduction in any medium or format, as long as you give appropriate credit to the original author(s) and the source, provide a link to the Creative Commons licence, and indicate if changes were made. The images or other third party material in this article are included in the article's Creative Commons licence, unless indicated otherwise in a credit line to the material. If material is not included in the article's Creative Commons licence and your intended use is not permitted by statutory regulation or exceeds the permitted use, you will need to obtain permission directly from the copyright holder. To view a copy of this licence, visit http://creativecommons.org/licenses/by/4.0/.

\section{References}

Andreoletti, G. (2019). Fatalism and future contingents. Analytic Philosophy, 60(3), 1-14.

Baron, S. (2017). Back to the unchanging past. Pacific Philosophical Quarterly, 98(1), 129-147.

Bernstein, S. (2017). Time travel and the movable present. In Keller, J. A. (Ed.), Being, Freedom, and Method: Themes from the Philosophy of Peter van Inwagen (pp. 80-94). Oxford University Press.

Cameron, R. (2015). The moving spotlight: an essay on time and ontology. Oxford University Press.

Chisholm, R. (1966). Freedom and action. In Lehrer, K. (Ed.), Freedom and Determinism (pp. 11-44). Random House.

Clarke, R. (1993). Toward a credible agent-causal account of free will. Noûs, 27(2), 191-203.

Goddu, G. C. (2003). Time travel and changing the past. Ratio, 16(1), 16-32.

Goddu, G. C. (2011). Avoiding or changing the past? Pacific Philosophical Quarterly, 92(1), 11-17. 
Geach, P. T. (1977). Providence and Evil. Cambridge University Press.

Goldblatt, R. (1992). Logics of Time and Computation ( $2^{\text {nd }}$ ed.). CSLI Publications.

Griffith, M. (2013). Free Will: The Basics. Routledge.

Hudson, H. \& Wasserman, R. (2010). Van Inwagen on time travel and changing the past. In Zimmerman, D. (Ed.), Oxford Studies in Metaphysics, Vol. 5 (pp. 41-49). Oxford University Press.

Koymans, R. (1990). Specifying real-time properties with metric temporal logic. Real-Time Systems, 2(4), 255-299.

Law, A. (2019). The puzzle of hyper-change. Ratio, 32, 1-1.

Lewis, D. (1976). The paradoxes of time travel. American Philosophical Quarterly, 13, 145-152.

Markosian, N. (1999). A compatibilist version of the theory of agent causation. Pacific Philosophical Quarterly, 80(3), 257-277.

Meiland, J. W. (1974). A two-dimensional passage model of time for time travel. Philosophical Studies, 26(3-4), 153-73.

O'Connor, T. (1995). Agent causation. In O'Connor, T. (Ed.), Agents, causes, and events (pp. 61-79). Oxford University Press.

O'Connor, T. (2001). Dualist and agent-causal theories. In Kane, R. H. (Ed.), The Oxford Handbook of Free Will (pp. 337-355). Oxford University Press.

Pezet, R. E. (2017). Against non-Ludovician time. Analytic. Philosophy, 58(4), 330-59.

Prior, A. N. (1996). Some free thinking about time. In Copeland, B. J. (Ed.), Logic and Reality: Essays on the Legacy of Arthur Prior (pp. 45-46). Oxford University Press.

Spolaore, G., \& Gallina, F. (2020). The actual future is open. Erkenntnis, 85(1), 99-119.

Spolaore, G., \& Torrengo, G. (2019). The Moving Spotlight(s). Inquiry, Online first. https://doi.org/10. 1080/0020174X.2019.1610046.

Todd, P. (2011). Geachianism. In Kvanvig, J. L. (Ed.), Oxford Studies in Philosophy of Religion, Vol. 3 (pp. 222-251). Oxford University Press.

Todd, P. (2016). On behalf of a mutable future. Synthese, 193(7), 2077-2095.

Todd, P., \& Rabern, B. (2021). Future contingents and the logic of temporal omniscience. Noûs, 55(1), $102-127$.

Wasserman, R. (2017). The Paradoxes of Time Travel. Oxford University Press.

Publisher's Note Springer Nature remains neutral with regard to jurisdictional claims in published maps and institutional affiliations. 